\title{
Compliance With Guideline of Hypertension Management Among Health Practitioners in Illubabor and Buno Bedelle Zones, South West Ethiopia; 2020
}

Gebremeskel Mulatu

Mettu University

Daniel Mengistu

Addis Ababa University

Bizuayehu Atinafu Ataro ( $\nabla$ bizuayehu.atinafu@aau.edu.et )

Wolaita Sodo University

\section{Research Article}

Keywords: Compliance, hypertension, guideline and Health practitioners

Posted Date: January 21st, 2022

DOI: https://doi.org/10.21203/rs.3.rs-1179879/v1

License: (c) (1) This work is licensed under a Creative Commons Attribution 4.0 International License.

Read Full License 


\section{Abstract}

Background: To achieve sustainable development goal and to improve management of noncommunicable diseases the federal democratic republic of Ethiopian ministry of health developed guidelines on clinical and programmatic management of major non communicable diseases in 2016. Compliance to hypertension guideline was very low in developing countries leading to compromised quality of life and premature deaths. The aim of this study was to assess health practitioners' compliance with hypertension management guideline and its associated factors in Illubabor and Buno Bedelle zones.

Method: A cross-sectional study design from patient medical record data was used to assess for compliance with hypertension management guideline. Medical records of adult Patients treated for hypertension from March 2019 to march 2020 was reviewed. Simple random sampling method was used to select the first study population then systematic sampling was used to select consecutive study populations. The collected data was checked for its completeness, consistency and accuracy before analysis. Data was coded, entered and cleaned using Epi- data 7 and export to SPSS version 25 for analysis. Multivariate analysis was used to obtain a final model describing the significant independent predictors of guidelines compliance.

Results: overall compliance of health practitioners' to hypertension guideline was found to be poor; only $75(19.5 \%)$ patients were managed following the guideline. In multivariate analysis, availability of resource (adjusted odds ratio $[\mathrm{AOR}]=0.400,95 \% \mathrm{Cl}:=0.203-0.788$ ), supervision status (adjusted odds ratio $[\mathrm{AOR}]=0.497,95 \% \mathrm{Cl}:=0.308-0.892$ ), training status (adjusted odds ratio $[\mathrm{AOR}]=0.250,95 \% \mathrm{Cl}$ : $=0.107-0.584$ ), and appointment status (adjusted odds ratio $[A O R]=0.091,95 \% \mathrm{Cl}:=0.024-0.352$ ) had significant positive association with compliance to hypertension management guideline.

Conclusion: Only $75(19.5 \%)$ of patients were managed following the guideline; which indicates nearly more than two third of patients were not managed following the national hypertension guideline, primarily due to a lack of recommending lifestyle modifications.

\section{Background}

Hypertension (HTN) is among cardiovascular Non-communicable diseases (NCDs) that are noncontagious diseases with long duration; which takes a high percentage of overall mortality, and known to push many people into poverty due to catastrophic spending on medical care (1). NCDs are embedded in sustainable development goal (SDG) target 3.4 , that is, to reduce by one-third the premature mortality from non-communicable diseases by 2030(2). To meet SDG and to enhance management of NCDs the federal democratic republic of Ethiopia ministry of health (FDREMOH) developed Guidelines on Clinical and Programmatic Management of Major Non Communicable Diseases including HTN in 2016 (3). There's global consensus that using the primary health-care system, which provides a decentralized and integrated platform of care is vital in addressing non-communicable diseases (4); that is why the 
FDREMOH have adapted the world health organization (WHO) package to the local context and developed the guideline for primary health care unit use. These guidelines deal in a comprehensive manner with all realms of NCDs interventions like prevention, treatment and care. The main audience comprises nurses, health officers and general practitioners (MD) performing at the first care level, especially health centers and primary hospitals (3). Primary health care (PHC) was defined as health facilities that serve as the first point of contact for ill patients but excluded inpatient hospital settings (5).

A guideline is any document containing recommendations about health interventions, whether these are clinical, public health or policy recommendations. A recommendation provides information about what policy-makers, health-care providers or patients should do. It implies a choice between different interventions that have an impression on health care and have implication on utilization of resources. Guidelines are recommendations intended to help providers and recipients of health care and other stakeholders to form informed decisions in order to improve patient outcome. Guideline can be: 1) A rapid advice guideline which are produced in response to a public health emergency during which WHO is required to supply rapid global leadership and guidance, 2 ) a standard guideline that produced in response to an invitation for guidance in reference to a change in practice or controversy during a single clinical or policy area. 3) A compilation of guidelines contains current recommendations from WHOs and other sources, but doesn't include any new recommendations. 4) Full guideline is a guideline that gives complete coverage of a health topic or disease. (6). Guideline is also a summary of Major study findings which can easily be accessed by practitioners who are too busy to revise the most recent information from major trials. Many practitioners are not aware of these guidelines and a few are aware of them but are not compliant with them (7).

Eventhough non communicable diseases are mostly preventable, they account for 52.8 million deaths, and $54 \%$ of disability adjusted life years worldwide; Globally, among all NCDs, HTN accounts for $0.7 \%$ of the entire disability-adjusted life years (DALYs)(8). Contrary to public opinion, available data demonstrated that almost about $48 \%$ of NCDs in low and middle income countries (LMICs) occur among people under the age of 70, compared to twenty-eight percent that found in high-income countries (HICs) (9). Further, by the year 2020, global anticipated NCDs burden will rise to $80 \%$ and majority of deaths (70\%) will occur in LMICs; Many factors are identified as possible causes of the increased morbidity and mortality; this includes Demographic transition, adoption of modern trends of living, poor patient understanding of the disease process, and non adherence with prescribed medical regimens (10). Similarly, the magnitude of NCDs is increasing in Ethiopia (11). WHO, 2018 estimation shows that 39\% of Ethiopian population is dying from NCDs, with a national cardiovascular disorder prevalence of 16\% (12). This WHO estimation shows a rise of $1 \%$ in cardiovascular prevalence, since 2011 estimation(13). A study in Ethiopia showed the general prevalence of hypertension among adult population to be $27.9 \%$, with the proportion being $30.7 \%$ in urban and $25.3 \%$ in rural residents. In terms of sex proportion, $29.3 \%$ was for females and $26.3 \%$ for males (14). Prevalence of Hypertension (HTN) in Illubabor zone was found to be $14.2 \%$ (15). Whereas in Bedelle town, reported use of anti-hypertensive medication was $16.9 \%$ and the overall control rate of HTN was only $22.4 \%$ (16). Compliance has been defined as "the extent to which a person's behavior, corresponds with agreed recommendations and follows list of tasks given by 
some party." There is mounting evidence that non-compliance to the treatment guideline is prevalent across the world and is associated with adverse health outcomes, increased incidence of drug resistance, and higher costs of care (17). Lower risk of long-term morbidity and mortality, improved quality of life, and lower healthcare costs are some of the advantages of complying to guidelines shown in several studies (18). Studies showed that health practitioners' lack the tendency to follow the HTN guideline and prescribe more expensive drugs without evidence of efficacy (19). A study conducted in Bangladesh revealed that, compliance of health practitioners' to national guideline for HTN screening was weak (20). A population based study conducted in Sub-Saharan Africa showed that $40 \%$ of individuals with BP above the normal range are diagnosed as hypertensive, and among $30 \%$ of diagnosed cases which were on treatment, for only $20 \%$ of them BP was returned to the defined normal range(21). Studies found that there were substantial gaps between the development and dissemination of guidelines and their implementation in practice; specifically there was no change in health personnel attitude to follow guidelines(22)(23). A study have concluded that health personnel compliance to HTN guidelines has been low (24). An estimated $40 \%$ of 50 million persons within USA with HTN remain untreated, and $66 \%$ of hypertensive patients have BP values that are not controlled to the recommended levels (25). Complying with guideline and translating into concrete practice may be a complex process in which health practitioner-related, patient-related, guideline related, and training related factors all play a role (26). Some studies are conducted to show the extent to which knowledge of guideline recommendations for diagnosis and treatment affects how health practitioners' manage their patients (27). The German follow-up of the Hypertension Evaluation Project clearly demonstrated inadequate knowledge of the diagnosis and treatment of arterial hypertension (28). Observational studies have shown that the health care practitioners' attitudes, behavior towards hypertension management and deviation from the clinical practice guidelines account for $66 \%$ of poor control of hypertension (29). There's also evidence of deficiencies in treatment quality appear due to inadequate implementation of existing treatment recommendations (30). Generally, several studies are conducted across the world, most of them aren't recent, and only a few of this studies showed couples of things affect compliance of health practitioners' with HTN guideline. Moreover, there's no study documented about health practitioners' compliance to the 2016 national NCD guideline in the management of hypertension and its associated factors in Ethiopia. The aim of this study was to assess the health practitioners' compliance with HTN management guideline and its associated factors in Illubabor and Buno Bedelle zones.

\section{Objective}

To determine the prevalence of hypertension management guideline compliance among healthcare practitioners. To identify the factors associated with compliance of hypertension management guideline among healthcare practitioners.

\section{Methods}

\section{Study Area}


The study was conducted at four governmental health care units found in Illubabor and Buno Bedelle zones. One health center from Metu which is an administrative town of Ilu-Ababora Zone in South West Ethiopia, Oromia region which is located $600 \mathrm{~km}$ from the capital city of Ethiopia, Addis Ababa. Mettu, located in the Illubabor Zone of the Oromia Region along the Sor River, this town has a latitude and longitude of $8^{\circ} 18^{\prime} \mathrm{N} 35^{\circ} 35^{\prime} \mathrm{E}$ and an altitude of $1605 \mathrm{~m}$. Another one health center was included in the study from Gore town which located $18 \mathrm{Kms}$ away from mettu town. In addition, two governmental health care units from Bedelle town were also part of the study. Bedelle town is located in the south west of Ethiopia, $480 \mathrm{Km}$ from the capital Addis Ababa. The town have one general hospital, one health center and few private medium clinics. In all selected health care units, mostly nurses and health officers and rarely general practitioners have been trained on the HTN guideline and they were working at their respective Outpatient Departments $(15,16)$.

\section{Study Design and Period}

A retrospective cross sectional study design was conducted from March to April/2020 in Illubabor and Buno Bedelle zones, Oromia regional state, south west Ethiopia.

\section{Source Population}

All medical records of hypertension patients treated from March, 2019 to March, 2020.

\section{Study Population}

All sampled medical records of HTN patients who are treated in the health care units.

\section{Study variables}

Dependent variable: - Compliance to HTN management guideline.

Independent variables:- Supervision, Health Care unit level, Resources/supplies, Year of service, professional background, Qualification, Training, Attitude of the health practitioners' towards HTN guideline, Knowledge of the guideline and coo morbid cases

\section{Eligibility}

Inclusion Criteria:-Medical records of adult Patients ( $>18$ years old) treated for hypertension between March 1st, 2019 and March 1st, 2020

Exclusive Criteria: - Criteria for exclusion were medical records with missing information at first or consecutive follow up, and those for which inadequate information was available to establish whether appropriate treatment was received.

\section{Sample size determination}


The sample size for this study was calculated using a single population proportion formula based on the following assumptions:

\section{$\mathbf{n}=\underline{\left(\mathbf{Z}_{\alpha / 2}\right)^{2} \mathbf{P}(\mathbf{l}-\mathbf{P})}$}

(d) ${ }^{2}$

Where: $\mathbf{n}$-minimum sample size required for the study

d-Margin of error $=0.05$

$Z_{a / 2}$-Value of standard normal distribution ( $\left.Z=1.96\right)$ with confidence interval of $95 \%$ and $a$ is 0.05

$$
\mathrm{n}=\frac{\left(\mathrm{Z}_{\alpha / 2}\right)^{2} \mathrm{P}(1-\mathrm{P})}{(\mathrm{d})^{2}}=\frac{(1.96)^{2} 0.519(1-0.519)}{(0.05)^{2} 383.605 \approx 384}
$$

For the sample size calculation, the prevalence of compliance to hypertension management guideline was obtained from a previous study conducted in South Africa that found $51.9 \%$ to be overall compliance to HTN guideline(40).

\section{Sampling technique}

First, total sample size (384) was estimated based on the total number of HTN patients visited the governmental primary care units in 2019. Next the determined sample was proportionally allocated to each health units as follows Bedelle hospital $=120$, Bedelle $H C=84$, Gore $H C=60$, Mettu $H C=120$. Finally, simple random sampling method was used to select the first study population then systematic sampling was used to select consecutive study population.

\section{Data collection technique and instrument}

A checklist was created using the National HTN guideline consensus points to assess for compliance with the recommendations and questionnaire was adopted from previous similar studies to assess factors associated with primary care practitioners' compliance with guidelines on the basis of the existing published recommendations for the diagnosis, treatment, and other activities of HTN(3)(33). Data was collected by principal investigator. Since associated factors cannot be assessed from medical records, first primary data was collected from health practitioners' so that the specific time period they were in the NCD/HTN OPD and specific patients treated could be known and traced on medical record of patients. The questioner includes Health personnel biographic data(age, sex, specialization, experience, training on HTN guideline, availability of resource, health care unit, qualification and others); in order to avoid recall bias they were requested to check the health units 12 months schedule, and they were also requested to fill the questioners' on spot. The medical record of each patient was traced on the hospital/health centers Health management information system (HMIS) registration book following the specific time period allocated by the health practitioners'. Information from medical records include: patients General 
information, date at which the pharmacological treatment was initiated by the specific health practitioners', appointment status, date of consecutive follow-up, Data on blood pressure from the first and second appointments, presence of co morbidities, classification of disease given by the health practitioners', prescribed antihypertensive medication, and if any recommendation of lifestyle modification was made. Once the medical records were identified, the medical record number (MRN) was collected and a study identification number (Study ID) was assigned to each clinical chart so that similar patient's card will be excluded if appeared on another health practitioner' review (even if a patient is seen by more than one health practitioners', it was reviewed only for one health practitioner). The study identification number went from 001 to 384 . This code was stored in electronic format, encrypted using the encryption software Mac OS X version 10.9.8, and password-protected on the principal investigator's personal computer. Only the principal investigator has access to this file and to the personal computer where it is stored. No other identifier was collected, such as name, participant's home address.

\section{Data quality assurance}

To assure high quality of the data, emphasis was given in designing data collection instrument. In order to test reliability and assure the quality of the data, pretest of approximately $15 \%$ (maximum number was thought to yield a more accurate result) of the sample was performed. The following variables were tested: age, systolic and diastolic blood pressures during the first and second appointments, classification by health practitioners', lifestyle modification recommendations during first appointment, medications prescribed in the first appointment and existing co morbidities.

The co author did the pretest and these data were compared with the data collected by the principal investigator using the same clinical charts. To assess the level of agreement between the two abstractors, a kappa statistic was used for categorical variables and the intra class correlation coefficient for continuous variables. The kappa coefficient ranged from 0.854 to 1.000 and the intra class correlation coefficient ranged from 0.940 to 0.996 , showing very good to excellent agreement between pre testers.

\section{Data processing and analysis}

Data were coded, entered and cleaned using Epi- data 7 and export to SPSS version 25 for analysis. In the univariate analysis, for continuous variables Q-Q plots were used to assess if the variable was normally distributed. For those variables which were normally distributed, the mean and standard deviation were used as descriptive statistics; and for those that were not normally distributed the median and interquartile range (IQR) were used. For categorical variables, the results were presented as frequencies and proportions.

A bivariate analysis was conducted to compare independent variables with dependent variable. An independent sample $t$ test was used for continuous normally distributed variables, Wilcoxon Sum-Rank test for continuous non-normally distributed variables, and Fisher's exact test or chi-squared test for categorical variables, depending if the observed frequencies in any cell was less than five or not. 
Multivariate analysis was used to obtain a final model describing the significant independent predictors of guidelines compliance. All those variables which had statistically significant association with HTN guideline compliance in bivariate analysis were included in multivariate model. P-value and $95 \% \mathrm{Cl}$ for OR was used in judging the significance associations. P-value less than 0.05 were taken as significant association. The finding was presented by using, graphs and tables.

\section{Operational definition}

Compliance to HTN guideline-is an application of the strategy or the guideline according to the recommended manner. The level of compliance can be categorized as high level, and low level(40)

Health practitioners'- are those whose professional backgrounds are nurses, health officers, and general practitioners' involved on HTN management.

Supplies\resources- are equipment's used in the management of hypertension including the printed guideline, presence of separate NCD/HTN outpatient department, laboratory, medications, BP cuff etc.

Compliance-ability of health practitioners to follow NCD guideline of HTN assessment, diagnosis, treatment process and all activities listed on the guide line by FDREMOH.

\section{High level compliance:}

Correct implementation of recommended strategy for above $50 \%$ of patients

\section{Low level compliance:}

Below 50\%: correct implementation

The knowledge evaluating portion of the questionnaire consisted of 12 multiple-choice questions. A score of " 1 " point will be credited to each correct answer and " 0 " to each wrong answer and unanswered question.

Level of knowledge will be indicated as:

0-4 correct answer is Poor knowledge

5-8 correct answer is reasonable

9 and more correct answer is good

Attitude evaluation portion, consisting of 6 items, will be developed on the basis of extensive literature review(38). These items will be based on a 5-point Likert scale ranging from "Strongly Disagree" to "Strongly Agree" and scored as:

Strongly disagree $=1$, Disagree $=2$, Undecided $=3$, Agree $=4$, and strongly agree $=5$. 
Negative items were scored reversely, so that the high score reflects more positive attitude.

\section{Result}

\section{Univariate Analysis- patients' clinical characteristics}

Table 1 shows patients' clinical characteristics. Among the patients, $97(25.3 \%)$ of them were diagnosed as having hypertension for the very first time, whereas $287(74.7 \%)$ were on follow up treatment. For hypertension classification, $68(17.7 \%)$ of participants were classified as Stage II, 12(3.1\%) classified as Stage I, 29(7.6\%) were classified as having hypertensive crisis and $275(71.6 \%)$ were classified using other classifications like mild-moderate and controlled- uncontrolled. During the first appointment, the mean systolic blood pressure (SBP) was $153.8 \mathrm{mmHg}( \pm 23.5)$, while the mean diastolic blood pressure (DBP) was $97.1 \mathrm{mmHg}( \pm 33.8)$. In the consecutive appointment, the mean SBP was $142.5 \mathrm{mmHg}( \pm 17.0)$ and the mean DBP was $89.9 \mathrm{mmHg}( \pm 9.9)$. For the number of co morbidities, $347(90.4 \%)$ had no co morbidities, $24(6.3 \%)$ had diabetes mellitus, while $13(3.4 \%)$ had stroke. For blood pressure status on the consecutive follow up; $142(37.0 \%), 185(48.2 \%), 57(14.8 \%)$ of them had uncontrolled, controlled and below target level which is $140 / 90$ of the guideline consecutively. On the other hand, Table 2 presents the treatment characteristics of the patients. Regarding the number of antihypertensive medication, $274(71.4 \%)$ of cases were prescribed with one medication, $78(20.3 \%)$ of cases were prescribed two antihypertensive medications and $10(2.6 \%)$ was prescribed with three medications while $22(5.7 \%)$ of them referred to higher facilities. Lifestyle modification recommendations were given to $79(20.6 \%)$ of participants, while they weren't given to $305(79.4 \%)$. The majority of health practitioners' complied with the pharmacological Antihypertensive treatment protocols for $238(62.0 \%)$ patients, while for $146(38.0 \%)$ of the patients the guideline were not followed. Combining the lifestyle modification recommendations and pharmacological antihypertensive treatment to assess compliance with the hypertension management guideline, only $75(19.5 \%)$ patients were managed following the guideline, while $309(80.5 \%)$ did not. 


\section{Characteristic}

First visit SBP, mmHg; Mean (SD)

First visit DBP, mmHg; Mean (SD)

Follow-up SBP, mmHg; Mean (SD)

Follow-up DBP, mmHg; Mean (SD)

Appointment status; $\mathrm{n}(\%)$

Initial

Follow up

Total

Disease stage on record; $n(\%)$

Pre hypertension

Stage I HTN

Stage II HTN

Hypertensive crisis

Other staging

Total

Co morbidities; n (\%)

None

DM

Stroke

total

BP Status; n (\%)

Uncontrolled

Controlled

Below target

Total

Note. $\mathrm{SD}=$ standard deviation; $\mathrm{n}$ = number of subjects; SBP, systolic blood pressure; DBP, diastolic blood pressure; HTN, hypertension; DM, diabetes mellitus. For continuous and normally distributed variables the mean was used as central tendency measure.
$153.8( \pm 23.5)$

$97.1( \pm 33.8)$

$142.5( \pm 17.0)$

$89.9( \pm 9.9)$

97(25.3)

287(74.7)

$384(100)$

0

12(3.1)

68(17.7)

29(7.6)

275(71.6)

384(100)

347(90.4)

24(6.3)

13(3.4)

384(100)

142(37.0)

185(48.2)

57(14.8)

384(100) 
Table 2

treatment characteristics of study participants

\section{Characteristics}

Number of Antihypertensive medication(s) prescribed; $\mathrm{n}(\%)$

1 medication prescribed

274(71.4)

2 medications prescribed

78(20.3)

3 medications prescribed

10(2.6)

Referred to higher facilities

22(5.7)

Total

$384(100)$

Lifestyle Modifications recommended; n (\%)

YES

79(20.6)

NO 305(79.4)

Total $384(100)$

Antihypertensive medication(s) following the guideline; $\mathrm{n}(\%)$

YES

NO

Total

Management compliance with HTN guideline;

n (\%)

YES

75(19.5)

NO

Total

$384(100)$

Note. $n=$ number of subjects; HTN= hypertension; the result of management compliance with HTN guideline was yielded by combining lifestyle recommendations and anti-hypertensive medications as explained on appendix Il; YES = for whom guideline recommendation was followed; $\mathrm{NO}=$ for whom guideline recommendations not followed.

\section{Biographic characteristics of Health practitioners'}

As shown on Table 3, there were more males 13(68.4\%) than females 6(31.6\%); and the mean age of the health practitioners' were 33.9 years $( \pm 7.8)$. Regarding professional background; $6(31.6 \%)$ were general practitioners' (GPs), 9(47.9\%) were health officers (HOs) and 4(21.1\%) of them were nurses. Qualification 
of health practitioners' were a first degree for all of them which named as:13(68.4\%) had BSc degree and $6(31.6 \%)$ of them were medical doctors and their mean year of clinical experience were 5.3 years $( \pm 3.3)$. From the health practitioners', most of; $13(68.4 \%)$ were enrolled in health centers whereas $6(31.6 \%)$ were in hospital; $12(63.2 \%)$ of them responded as having resources for management of hypertension and $7(36.8 \%)$ didn't. Among the study participants $7(36.8 \%)$ have received training on Hypertension guideline where $12(63.2 \%)$ were not trained. $5(26.3 \%)$ responded as having close supervision and $14(73.7 \%)$ had no supervision. 
Table 3

Demographic characteristics of the health practitioners'

\section{Age (years) Mean; ( $\pm S D)$}

$33.9( \pm 7.8)$

Sex; n (\%)

Male

Female

Total

Designation; $\mathrm{n}(\%)$

General practitioner

$6(31.6)$

Health officer

$9(47.9)$

Nurse

$4(21.1)$

Total

$19(100)$

Qualification; n (\%)

Medical doctors

$6(31.6)$

BSc

$13(68.4)$

Total

19 (100)

Year of experience; Mean $( \pm S D)$

$5.3( \pm 3.3)$

Training status; $\mathrm{n}(\%)$

Trained

Not trained

$12(63.2)$

Total

19 (100)

Work place; n (\%)

Health center

Hospital

6 (31.6)

Total

$19(100)$

Resource available; n (\%)

Yes

12 (63.2)

No

7 (36.8)

total

19 (100)

Note. SD = standard deviation; $\mathrm{n}$ = number of subjects; for continuous and normally distributed variables the mean was used as central tendency measure. 
Have supervision; $\mathrm{n}(\%)$

Yes

No

Total

Note. SD = standard deviation; $\mathrm{n}$ = number of subjects; for continuous and normally distributed variables the mean was used as central tendency measure.

\section{Health practitioners' knowledge, attitude, and compliance to HTN guideline}

Table 4

percentage of answers confirming to HTN guideline (2016) for knowledge assessing questions

\section{Question number}

n $\%$

(1) Which of the following blood pressure (BP) values defines hypertension in an adult subject without coo morbidities?

(2) The absolute risk of cardiovascular events over 10 years in high risk patients is?

(3) In newly diagnosed, uncomplicated hypertension and no compelling indications, all of the following antihypertensive drug classes are agents of choice of first line mono therapy except,

(4) Which are the BP values, that after repeated measurements, define elderly subjects (>75 years) as hypertensive?

(5) Which one of the following antihypertensive drug classes you would like to prescribe as first choice for hypertensive patient with angina?

(6) Which one of the following antihypertensive drug classes you would like to prescribe 10 as first choice for hypertensive patients with protein uric CKD?

(7) Which one of the following antihypertensive drug classes you would like to prescribe

as first choice for hypertensive patients with systolic heart failure? Choose that apply

(8) Which are the appropriate examinations to be prescribed for the minimum diagnostic work-up of hypertensive patients?

(9) Which is the definition of Stage 1 hypertension?

(10) Which of the following actions are wrong during BP measurement? Choose all that apply

(11) Which one of the following is NOT a reason that; after evaluating patients, primary healthcare providers should refer patients to higher levels of care?

(12) Which BP value is the goal of management for elderly subjects (>75 years)?

Note. $\mathrm{n}=$ number of subjects answered the questions correctly 
The percentages of correct answers to the 12 questions are shown in Table 4. The mean number of correct answers was $6.42 \pm 6.25$ (range 2-12). The absolute number of guidelines conforming answers in the total study population is shown in Figure 1. From the questions: two, four, five, and twelve questions were answered by $1(5.3 \%)$ health practitioner; while seven questions and eight questions were answered by $2(10.5 \%)$ health practitioners. Six questions and nine questions were answered correctly by $4(21.1 \%)$ study participants. Among the remaining 3(15.8\%) study participants answered three questions correctly and none of the study participants have answered less than two questions. The knowledge status of health practitioners in the total study participants is presented on Figure 2. Analysis of the replies to the questionnaire showed that similar proportion of the participants had good $5(26.3 \%)$ and poor $5(26.3 \%)$ knowledge of hypertension guideline/2016, whereas $9(47.4 \%)$ of them had reasonable knowledge.

Health practitioners' in the study were highly positive towards the HTN guideline (2016), with mean attitude score of $23.63 \pm 4.13$, ranging from 11 to 30 on a 30 -point scale. Health practitioners' responses to attitude statements are given in Table 5 . A total of $75(19.5 \%)$ patients were managed in compliance with guidelines. The mean compliance of health practitioners to hypertension management guideline was $3.95 \pm 4.76$ ranging from 0 to 14 per maximum of 21 patients observed per health practitioner

Table 5

Attitude towards and compliance to HTN guideline (2016)

\begin{tabular}{|llllll|}
\hline Statements & SD & D & UD & A & SA \\
\hline $\begin{array}{l}\text { I have trust in the recommendations and developing committee } \\
\text { of HTNG(2016) }\end{array}$ & 1 & - & - & 11 & 7 \\
HTNG(2016) on the management of hypertension is helpful for & 2 & - & - & 8 & 9 \\
Health practitioners' & 1 & 1 & 1 & 8 & 8 \\
Adherence to HTNG (2016) Would produce desired out come & - & - & 4 & 9 & 6 \\
HTNG(2016) is motivated by desire to cut cost & 6 & 6 & 3 & 4 & - \\
HTNG(2016) decreases doctors' autonomy & 3 & 6 & 5 & 5 & - \\
HTNG(2016) is too rigid to apply to individual patients & & & & \\
compliance to HTN guideline (2016) Mean ( \pm SD) & $3.95( \pm 4.76)$ & & & \\
\hline $\begin{array}{l}\text { Note: mean is used to show health practitioners' compliance to guideline as Mean }( \pm S D) ; ~ H T N= \\
\text { hypertension; SA=strongly agree; A=agree; UD=undecided; DA=disagrees=strongly disagree. }\end{array}$ \\
\hline
\end{tabular}

\section{Bivariate Analysis}

Health practitioners' compliance to Hypertension management guideline and health system related factors 
Table 6 presents the results of the bivariate analyses between the health practitioners' compliance to HTN management guideline and health system related factors. Those health practitioners' who were working at health center were more compliant $(4.4, \pm 5.3)$ than those working at hospital $(3.0, \pm 3.5)$. However, this difference was not statistically significant $(p=0.555)$. Among the study participants those who said they have close supervision were more compliant with mean compliance of $(4.0, \pm 4.8)$ than those who said they don't $(1.8, \pm 2.8)$; and this difference was statistically significant $(p \leq 0.001)$. Statistically significant difference have also seen $(p \leq 0.001)$ between health practitioners' who said resources that needed for hypertension management are available and those who said resource not available; those with available resources were more compliant $(5.6, \pm 5.1)$ than those with no resource available $(1.1, \pm 2.3)$.

Table 6

Compliance to hypertension management guideline and health system factors

\begin{tabular}{|c|c|c|}
\hline \multirow[t]{2}{*}{ Variables } & Compliance to HTNG (2016) & $P$ value* \\
\hline & \multicolumn{2}{|l|}{ Mean $( \pm S D)$} \\
\hline \multicolumn{3}{|c|}{ Resource available } \\
\hline Yes $^{a}$ & $5.6( \pm 5.1)$ & 0.049 \\
\hline No & $1.1( \pm 2.3)$ & \\
\hline \multicolumn{3}{|l|}{ Supervision } \\
\hline Yes $^{a}$ & $4.0( \pm 4.8)$ & 0.001 \\
\hline No & $1.8( \pm 2.8)$ & \\
\hline \multicolumn{3}{|l|}{ Work place } \\
\hline Health center ${ }^{a}$ & $4.4( \pm 5.3)$ & 0.555 \\
\hline Hospital & $3.0( \pm 3.5)$ & \\
\hline \multicolumn{3}{|c|}{$\begin{array}{l}\text { Note. HTNG = hypertension management guideline; } S D=\text { standard deviation; P-values were obtained } \\
\text { by using the chi-square test for categorical variables. Mean }( \pm S D) \text { was used to express compliance } \\
\text { status of health practitioners'; }{ }^{\prime}<.05 .{ }^{\text {a }} \text { Reference group. There were no missing data during the } \\
\text { analysis }(n=19) \text {. }\end{array}$} \\
\hline
\end{tabular}

\section{Compliance to Hypertension management guideline and health practitioners' related factors.}

Table 7 presents Compliance to Hypertension management guideline and health practitioners' related factors. Those whose age is $>30$ have shown more compliance to hypertension management guideline than those $\leq 30$ with mean compliance $4.0( \pm 4.8)$ and $3.9( \pm 4.7)$ respectively. But the difference seen between these age groups were not statistically significant $(p=0.967)$. Male health practitioners' had better compliance $(4.2, \pm 4.5)$ to the guideline in managing hypertensive patients than their female partners. However, this difference was not statistically significant $(p=0.781)$. 
Even though study groups with $<2$ years of clinical experience shown better compliance $(4.6, \pm 8.0)$ than 24 years' experience $(4.5, \pm 3.9)$ who complied more than who have $5-8$ years of clinical experience $(3.9$, $\pm 5.3)$ than those with $>8$ years $(2.3, \pm 3.2)$, Statistically significant difference was not observed $(p=0.521)$ between compliance these groups.

When we compare health practitioners' compliance with their professional background, Nurses have shown more compliance $(8.8, \pm 6.2)$ to the guideline than their counter general practitioners $(3.0, \pm 3.5)$ and health officers. But the difference between the study groups was not statistically significant $(p=0.094)$. Health practitioners' with BSc qualification were more compliant $(4.4, \pm 5.3)$ compared to Medical doctors whose mean compliance was $(3.0, \pm 3.5)$. And again the difference between them was not statistically significant $(p=0.555)$.

Among study participants, those who had training on the guideline have better compliance $(8.7, \pm 3.7)$ than their counter untrained parts $(1.1, \pm 2.3)$; and the difference between these groups have shown statistical significance $(p \leq 0.001)$. Study participants with good knowledge have better compliance (5.2, $\pm 5.4)$ than those who have poor knowledge of guideline and better compliance $(4.8, \pm 6.1)$ than who have reasonable knowledge whose compliance was $(2.8, \pm 3.8)$. However the difference between these groups were not statistically significant $(p=0.894)$. Health practitioners with total attitude score of $\leq 20$ have more $(6.5, \pm 3.5)$ compliance than whose total attitude score was $>25(4.4, \pm 5.9)$ whose compliance was better than those who had total attitude score of $21-25(3.3, \pm 4.6)$. But the difference between these study groups have no statistical significance $(p=0.815)$. 
Table 7

compliance to hypertension management guideline and health practitioners' related factors

\begin{tabular}{|c|c|c|}
\hline \multirow[t]{2}{*}{ Variables } & Compliance to HTNG (2016) & $P$ value* \\
\hline & \multicolumn{2}{|l|}{ Mean ( $(\mathrm{SD})$} \\
\hline \multicolumn{3}{|l|}{ Age } \\
\hline$\leq 30$ & $3.9( \pm 4.7)$ & 0.967 \\
\hline$>30^{a}$ & $4.0( \pm 4.8)$ & \\
\hline \multicolumn{3}{|l|}{ Sex } \\
\hline Male $^{a}$ & $4.2( \pm 4.5)$ & 0.781 \\
\hline Female & $3.5( \pm 5.6)$ & \\
\hline \multicolumn{3}{|c|}{ Year of clinical experience } \\
\hline$<2$ years $^{a}$ & $4.6( \pm 8.0)$ & 0.521 \\
\hline $2-4$ years & $4.5( \pm 3.9)$ & \\
\hline 5-8 years & $3.9( \pm 5.3)$ & \\
\hline$>8$ years & $2.3( \pm 3.2)$ & \\
\hline \multicolumn{3}{|c|}{ Professional background } \\
\hline GP & $3.0( \pm 3.5)$ & 0.094 \\
\hline Health officer & $2.4( \pm 3.7)$ & \\
\hline Nurse ${ }^{a}$ & $8.8( \pm 6.2)$ & \\
\hline \multicolumn{3}{|l|}{ Qualification } \\
\hline $\mathrm{BSc}^{\mathrm{a}}$ & $4.4( \pm 5.3)$ & 0.555 \\
\hline MD & $3.0( \pm 3.5)$ & \\
\hline \multicolumn{3}{|c|}{ Training status } \\
\hline Not trained & $1.1( \pm 2.3)$ & 0.001 \\
\hline Trained $^{\mathrm{a}}$ & $8.7( \pm 3.7)$ & \\
\hline
\end{tabular}

Note. $\mathrm{HTNG}=$ hypertension management guideline; $\mathrm{GP}=$ general practitioners; $\mathrm{MD}=$ Medical doctors $\mathrm{SD}=$ standard deviation; P-values were obtained by using the chi-square test for categorical variables, $t$-test for continuous variable. Mean $( \pm S D)$ was used to express compliance status of health practitioners'; $; 0.05$. ${ }^{a}$ Reference group. There were no missing data during the analysis $(n=19)$. 


\begin{tabular}{|c|c|c|}
\hline \multirow[t]{2}{*}{ Variables } & Compliance to HTNG (2016) & $P$ value* \\
\hline & \multicolumn{2}{|l|}{ Mean ( \pm SD) } \\
\hline Poor knowledge & $4.8( \pm 6.1)$ & \multirow[t]{3}{*}{0.894} \\
\hline Reasonable knowledge & $2.8( \pm 3.8)$ & \\
\hline Good knowledge a & $5.2( \pm 5.4)$ & \\
\hline \multicolumn{3}{|l|}{ Attitude towards HTNG } \\
\hline$\leq 20^{a}$ & $6.5( \pm 3.5)$ & \multirow[t]{3}{*}{0.815} \\
\hline $21-25$ & $3.3( \pm 4.6)$ & \\
\hline$>25$ & $4.4( \pm 5.9)$ & \\
\hline \multicolumn{3}{|c|}{$\begin{array}{l}\text { Note. HTNG= hypertension management guideline; GP= general practitioners; } M D=\text { Medical doctors } \\
\text { SD = standard deviation; } P \text {-values were obtained by using the chi-square test for categorical variables, } \\
\text { t-test for continuous variable. Mean }( \pm S D) \text { was used to express compliance status of health } \\
\text { practitioners'; } ;{ }^{\prime}<.05 .{ }^{a} \text { Reference group. There were no missing data during the analysis }(n=19) \text {. }\end{array}$} \\
\hline
\end{tabular}

\section{Health practitioners' compliance and patient related factors}

Table 8 shows health practitioners' compliance to hypertension management guideline and patient related factors. Health practitioners have shown better compliance $(0.2, \pm 0.4)$ when they have treated patients with hypertension only and patients with stroke than when they have treated patients with diabetes mellitus $(0.1, \pm 0.3)$. However this difference have no statistical significance $(p=0.817)$. Patients who have been on their first visit to the health practitioners' were treated more compliantly $(0.4, \pm 0.5)$ than those who were on their follow up visit $(0.1, \pm 0.3)$. And this difference has shown statistical significance of $(p \leq 0.001)$. 
Table 8

health practitioners' compliance to hypertension management guideline and patient related factors.

\begin{tabular}{|c|c|c|}
\hline Variables & Compliance HTNG (2016) Mean ( \pm SD) & $P$ value* \\
\hline \multicolumn{3}{|l|}{ Co morbidities } \\
\hline No co morbidities ${ }^{a}$ & $0.2( \pm 0.4)$ & 0.817 \\
\hline HTN+DM & $0.1( \pm 0.3)$ & \\
\hline HTN+STROKE & $0.2( \pm 0.4)$ & \\
\hline \multicolumn{3}{|l|}{ App status } \\
\hline First visit ${ }^{a}$ & $0.4( \pm 0.5)$ & $\leq 0.001$ \\
\hline Follow-up & $0.1( \pm 0.3)$ & \\
\hline
\end{tabular}

\section{Multivariate analysis}

Table 9 shows result of multivariate analysis of significant factors of hypertension management guideline compliance. In this model, availability of resource (adjusted odds ratio [AOR] $=0.400,95 \% \mathrm{Cl}$ : $=0.203-0.788$ ), supervision status (adjusted odds ratio $[\mathrm{AOR}]=0.497,95 \% \mathrm{Cl}:=0.308-0.892$ ), training status (adjusted odds ratio $[\mathrm{AOR}]=0.250,95 \% \mathrm{Cl}:=0.107-0.584$ ), and appointment status (adjusted odds ratio $[\mathrm{AOR}]=0.091,95 \% \mathrm{Cl}:=0.024-0.352)$ had significant positive association with compliance to hypertension management guideline. 
Table 9

multivariate analysis of factors of hypertension management guideline compliance

\begin{tabular}{|llllll|}
\hline Variables & B & SE & P value & AOR & 95\% Cl \\
\hline Resource available & 0.915 & 0.346 & 0.018 & 0.400 & $0.203-0.788$ \\
\hline Ref=yes & & & & & \\
\hline $\begin{array}{l}\text { Supervision } \\
\text { Ref=yes }\end{array}$ & 0.725 & 0.138 & 0.038 & 0.497 & $0.308-0.892$ \\
\hline $\begin{array}{l}\text { Training status } \\
\text { Ref=trained }\end{array}$ & 0.288 & 0.764 & 0.036 & 0.250 & $0.107-0.584$ \\
\hline $\begin{array}{l}\text { App status } \\
\text { Ref=initial }\end{array}$ & 2.394 & 0.688 & 0.001 & 0.091 & $0.024-0.352$ \\
\hline $\begin{array}{l}\text { Note: AOR= adjusted odds ratio Cl=confidence interval; B=beta; SE=standard error; App stat= } \\
\text { appointment status; ref=reference group .Only statistically significant results are given in the table. }\end{array}$ \\
\hline
\end{tabular}

\section{Discussion}

In this study overall compliance of health practitioners' to FDREMOH HTN guideline were found to be poor; only $75(19.5 \%)$ of patients were managed following the guideline. This finding is lower than a finding from study in Malaysia which similarly revised medical record of patients and found $67.1 \%$ of them received guideline compliant treatment (33). It's also lower than a finding of compliance to Malaysian clinical practice guideline which indicated that two third (73.5\%) of the entire treatment were in compliance with Clinical practice guideline(38), and fewer than the finding from South Africa with over all compliance of $51.9 \%$ (42). This poor compliance in the current study is because the study participants have very poor compliance to life style recommendations which is applied for only $79(20.6 \%)$ patients.

The mean compliance of health practitioners to hypertension management guideline was $3.95 \pm 4.76$ starting from 0 to 14 per maximum of 21 patients observed per health practitioner. This finding is similar to a study which find the median number of guidelines compliant prescriptions was 13 (range 5-20) per 20 patients seen per each health practitioner (48). From the study, 13(68.4\%) of the health practitioners' were males and the mean age of the health practitioners' were 33.9 years $( \pm 7.8)$. Regarding professional background; 6(31.6\%) were general practitioners' (GPs), 9(47.9\%) were health officers (HOs) and 4(21.1\%) of them were nurses; $13(68.4 \%)$ of them had BSc degree and $6(31.6 \%)$ of them were medical doctors and their mean year of clinical experience were 5.3years $( \pm 3.3)$. Among the health practitioners', $13(68.4 \%)$ of them were enrolled in health centers whereas $6(31.6 \%)$ were in hospital. $12(63.2 \%)$ health practitioners' had resources for management of hypertension; there were $7(36.8 \%)$ trained and $5(26.3 \%)$ health practitioners who had close supervision. These characteristics of the study participants is incomparable with other studies because of variation in methodology and characteristics of the study participants; 
which found mean age of the physicians to be $41.05 \pm 8.71$ years and 59 (73.8\%) were males 41 (51.2\%) of them to have practiced for more than 10 years (48).

In the study mean number of correct answers to knowledge assessing questions was $6.42 \pm 6.25$ (range $2-12$ ) which is better than a study that found mean score of correct answers to be 5.3 points(46). From the questions: Six questions (half of total), were answered correctly by $4(21.1 \%)$ study participants and none of the study participants have answered less than two questions; which was comparable to a study whose only $20.1 \%$ of the study population correctly answered at least six of the questions (44). This better score of knowledge status is related to training and because some of the guideline terms were similar to other international guidelines such as WHOs. In current study, similar proportion of the participants, $5(26.3 \%)$ had good and $5(26.3 \%)$ poor knowledge of hypertension guideline/2016, whereas $9(47.4 \%)$ of them had reasonable knowledge. However this finding is less than study which showed $40 \%$ of the physicians knew the guidelines adequately(45) and was much less than a study whose $73.07 \%$ of study populations had adequate knowledge of guidelines(33).

Most of health practitioners' in the current study showed positive attitude towards the HTN guideline (2016), with mean attitude score of $23.63 \pm 4.13$, ranging from 11 to 30 on a 30 -pointscale. They trust the guideline as well as its developers. Almost all of them believed that the guideline is useful for them and complying to it would produce best patients' outcomes. This health practitioners' attitude was very close to study from Malaysia, in which mean attitude score of $23.15 \pm 1.34$ was found(33).

According to this study finding, health practitioners' who were working at health center were more compliant but no significant association was found with compliance to the guideline. Having close supervision and resources that needed for hypertension management were also found to have better compliance than their counter parts on bivariate analysis. These factors also found to have positive association with the dependent variable; compliance to HTN guideline on multivariate analysis at (adjusted odds ratio $[\mathrm{AOR}]=0.497,95 \% \mathrm{Cl}:=0.308-0.892),([\mathrm{AOR}]=0.400,95 \% \mathrm{Cl}:=0.203-0.788)$ respectively.

Even though previous studies didn't show these factors have association with HTN guideline, it was expected that the better the health system, the better the outcome will be; and this finding shall be one of the strengths of the current study.

Advanced age $(>30)$ and male sex have also been found to comply more, with mean score of $4.0( \pm 4.8)$ and $4.2( \pm 4.5)$ respectively, with no statistical significance found at bivariate analysis. The reason of this finding can be advantage of having more numbers compared to their counter parts as explained at health practitioners' biographic characteristics.

The study also found study participants with less clinical years of experience and BSc qualification were seen to comply better than those with long duration of experience and medical doctors. This finding was inconsistent with study conducted in Hong Kong, which stated health practitioners' with higher qualifications and longer duration of practice performed better as compared to health practitioners' with 
lower qualifications(50). The reason to the contrary finding is that study from Hong Kong was a large number of study participants survey and in the current study there was again quantitative advantage of the complying study groups.

Previous study stated degree of compliance to guideline can be affected by their specialty and coo work(49); and in the current study nurses have shown more compliance $(8.8, \pm 6.2)$ to the guideline than their counter general practitioners $(3.0, \pm 3.5)$ and health officers. But the difference between the study groups was not statistically significant $(p=0.094)$.

Training was found to have positive effect on the health practitioners' compliance to HTN guideline in multivariate analysis (adjusted odds ratio $[\mathrm{AOR}]=0.250,95 \% \mathrm{Cl}:=0.107-0.584$ ). This factor was not also indicated in previous studies; but, it is obvious that training can bring such out comes as found in this study.

Study participants with good knowledge were found to have better compliance with the guideline in the management of hypertension. This finding is consistent with finding of study which Statistically significant correlation was observed between knowledge and practice scores(33). And this finding is against studies from Italy (45) (46).

Statistically no significant association was found between health practitioners' attitude and their compliance. Applying positive Attitudes can be influenced by many factors, such as their knowledge, past clinical experience, beliefs about guidelines, outcome expectations, peers' opinions, and guidelines characteristics (41).

Health practitioners' compliance to HTN guideline is found to have significant positive association with patients appointment status (adjusted odds ratio $[\mathrm{AOR}]=0.091,95 \% \mathrm{Cl}:=0.024-0.352$ ); in which patients on their initial visit found to receive compliant treatment. The reason is that most of health practitioners' tend to give life style recommendations to patients whom they have met for first time and didn't apply same for patients who are on second and consecutive visits. This justification was indicated on a table of patients' treatment characteristics.

Health practitioners have shown better compliance $(0.2, \pm 0.4)$ when they have treated patients who had hypertension only and hypertensive patients with stroke than when they have treated hypertensive patients with diabetes mellitus $(0.1, \pm 0.3)$. This finding is consistent to a study from Malaysia that showed Doctors poorly adhered to guidelines in hypertensive patients with diabetes (40). The reason is because patients with concurrent co morbidities of overlapping path physiological pathways and management like hypertension and cardiovascular disease are more likely to receive guidelines adherent management(31).

\section{Limitation of the Study}

The main limitation of this study is that, the results may not be generalizable to the general population because the study areas were not chosen by randomization; instead it was selected based on 
accessibility and the investigators interest. Sample size was also another reason for lack of generalizability since it was difficult to get large number of health practitioners', it was calculated for patients rather than calculating for the health practitioners' themselves.

\section{Conclusion}

Complying to guidelines were believed to reduce risk of long-term morbidity and mortality, improve quality of life, and bring lower healthcare costs (18)(19).

This is the first study in Ethiopia which assessed health practitioners' compliance to HTN guideline and its associated factors. Only $75(19.5 \%)$ of patients were managed following the guideline; which indicates nearly more than two third of patients were not managed following the FDREMOH hypertension guideline, primarily due to a lack of recommending lifestyle modifications. The majority of health practitioners' complied with the pharmacological antihypertensive treatment protocols for $238(62.0 \%)$ of the patients.

Even though several health system related, health practitioners' related and patient related factors had association with complying with HTN management guideline, availability of resource (adjusted odds ratio $[A O R]=0.400,95 \% \mathrm{Cl}:=0.203-0.788$ ), supervision status (adjusted odds ratio $[\mathrm{AOR}]=0.497,95 \% \mathrm{Cl}$ : $=0.308-0.892$ ), training status (adjusted odds ratio $[\mathrm{AOR}]=0.250,95 \% \mathrm{Cl}:=0.107-0.584$ ), and appointment status (adjusted odds ratio $[\mathrm{AOR}]=0.091,95 \% \mathrm{Cl}:=0.024-0.352$ ) were found to have significant positive association with compliance to hypertension management guideline, in multivariate analysis.

\section{Abbreviations}

ACEI - Angiotensin Converting Enzyme Inhibitors, CHD - Coronary Heart Disease, CPG- Clinical Practice Guideline, DALYs - Disability-Adjusted Life Years, DM - Diabetes Mellitus, FDREMOH - Federal Democratic Republic of Ethiopia Ministry of Health, GRC - Guidelines Review Committee , HC - Health Center, HDL-C High Density Lipoprotein Cholesterol, HICs - High-Income Countries, HTN - Hypertension, IRB - Institution Review Board, LDL-C - Low Density Lipoprotein Cholesterol, LMICs - Low and Middle Income Countries, NCDs - Non-Communicable Diseases, OPDs - Outpatient Departments, PHC - Primary Health Care, PHCU Primary Health Care Units, PHCWs - Primary Health care Workers, SBP - Systolic Blood Pressure, SDG Sustainable Development Goal.

\section{Declarations}

We, the undersigned, declare that this paper is our original work has never been presented in any University and we understand that plagiarism will not be tolerated and all directly quoted material has been appropriately referenced

\section{Ethical Approval}


Ethical approval was obtained from Addis Ababa university Institutional Review Board (IRB-AAU), college of health sciences, school of nursing and midwifery research committee evidenced by meeting number: 09/2012EC. Following the approval by IRB, official letter of co-operation was written to respected study area from department of nursing. The study was conducted after permission from concerned management bodies was taken. Written Informed consent was taken from health practitioners' and data was collected after permission. The study also used patient record data. Ideally, it was preferable to have informed consent from the subjects of the study, even if there is no direct contact with them, but it was difficult to get them. However, all the necessary measures have been taken to maintain and assure the privacy, confidentiality and all benefits of the patients. Charts were reviewed at separate room, no mention has made about the names of the patients and care providers or anything related to the study. Data have been treated confidentially as subject identified by number only, and all methods were carried out in accordance with relevant guidelines and regulations.

\section{Conflict Interest}

The authors declares they have no competing interests.

\section{Funding}

No funding was received

\section{Consent for Publication}

Not applicable

\section{Availability of Data and Materials}

The datasets generated and/or analysed during the current study are not publicly available for because of to prevent any kinds of misuse by public before publication but are available from the corresponding author on reasonable request.

\section{Author's Contributions}

GM and BAA: Conceive of data and designed the study, supervised the data collection, performed the analysis, interpretation of data, drafted the manuscript and final approval of the revision for publication. DM Assisted in designing the study, data interpretation and critically reviewed the manuscript.

\section{Acknowledgments}

The authors acknowledge Addis Ababa University, the supervisors; at last but not least the authors extend their appreciation to management and staff of our study area for their invaluable support to achieve this study.

\section{References}


1. Nugent R, Bertram MY, Jan S, Niessen LW, Sassi F, Jamison DT, et al. Series The Lancet Taskforce on NCDs and economics 1 Investing in non-communicable disease prevention and management to advance the Sustainable Development Goals. lancet. 2018;05(19;391(10134)):2029-35.

2. WHO. Health in 2015: from MDGs, millennium development goals to SDGs, sustainable development goals. [internet]. 2015;

3. Dagnaw WW, Yadeta D, Feleke Y, Kebede T. Ethiopian National Guideline on Major NCDs 2016 Guidelines on Clinical and Programmatic Management of Major Non Communicable Diseases. Addis Ababa: FDREMOH; 2016. 52-65 p.

4. Ruby A, Knight A, Perel P, Blanchet K, Roberts B. The Effectiveness of Interventions for NonCommunicable Diseases in Humanitarian Crises: A Systematic Review. Lancet. 2015;23:1-16.

5. S. G. is the declaration of Alma Ata still relevant to primary health care? Br Med J. 2008;336:536-8.

6. WHO. handbook for guideline development. NCBI.

7. Siko PR, van Deventer C. Compliance with standard treatment guidelines in the management of hypertension: A review of practice of healthcare workers in Potchefstroom, North West Province, South Africa. South African Fam Pract. 2017;59(2):72-7.

8. Arts DL, Voncken AG, Medlock S, Abu-Hanna A van WH. Reasons for intentional guideline nonadherence: A systematic review. Int J Med Inf. 2016;89:55-62.

9. Mendis, S., Puska, P., \& Norrving, B. (2011). Global Atlas on cardiovascular disease prevention and control. Geneva: World Health Organization.

10. Mulugeta $Y$, Id D, Firew BS. Factors associated with noncommunicable disease among adults in Mecha district, Ethiopia: A case control study. 2019;1-13.

11. Woldesemayat EM, Kassa A, Gari T, Dangisso MH. Chronic diseases multi-morbidity among adult patients at Hawassa University Comprehensive Specialized Hospital. BMC Public Health. 2018 Mar $14 ; 18(1)$.

12. WHO. Noncommunicable diseases country profiles 2018 . Geneva: 87 p.

13. WHO. Noncommunicable diseases country profiles 2 011. Geneva: 207 p.

14. Abebe SM, Berhane Y, Worku A, Getachew A. Prevalence and associated factors of hypertension: A crossectional community based study in Northwest Ethiopia. PLoS One. 2015;10(4):1-11.

15. Al. TS et. Magnitude and Associated Factors of Hypertension among Adults in Illubabore Zone of Oromia National Regional State, South West Ethiopia. Indo Am J P Sci. 2018;05(11):11549-55.

16. Feyie B, Esayas K, Gudina WH etal. Prevalence of Hypertension and Associated Factors in Bedele Town, Southwest Ethiopia. Ethiop J Heal Sci. 2014;1(24):21-6.

17. WHO. Adherence to long-term terapies. 2003;29(8):1228-33.

18. Saleh F, Mumu SJ, Ara F, Hafez A, Ali L. Non-adherence to self-care practices \& medication and health related quality of life among patients with type 2 diabetes: a cross-sectional study. 2014;14(1):1-8.

19. Huse DM, Roht LH, Alpert JS, Hartz SC. Physicians' knowledge, attitudes and practice of pharmacologic treatment of hypertension. Ann Pharmacother 2001;35:1173-9. 
20. Zaman MM, Nieveras $\mathrm{O}$, Talukder HK et al. Current health system scenario for addressing noncommunicable diseases in Bangladesh: Bangladesh health Watch report, 2016. non-communicable diseases in Bangladesh current scenario and future directions. Dhaka: James P Grant School of Public Health BRAC University; 2016. 43-55 p.

21. Addo J, Smeeth L, Leon DA. Global Health - Hypertension in Sub-Saharan Africa. A Systematic Review. 2007;1012-8.

22. Worrall G, Chaulk P, Freake D. The effects of clinical practice guidelines on patient outcomes in primary care: a systematic review. 1997;1705-12.

23. Milchak JL, Carter BL, James PA, Ardery G. Measuring adherence to practice guidelines for the management of hypertension: An evaluation of the literature. Vol. 44, Hypertension. 2004. p. 602-8.

24. Hicks LS, Fairchild DG, Horng MS, Orav EJ, Bates DW, Ayanian JZ. Determinants of JNC VI Guideline Adherence, Intensity of Drug Therapy, and Blood Pressure Control by Race and Ethnicity. 2004;

25. Chobanian A V, Bakris GL, Black HR, Cushman WC, Green LA, Izzo JL, et al. seventh report of joint national committe on prevention, detection and treatment of non communicable disease. 2003;1206-52.

26. Grimshaw JM, Thomas RE, MacLennan G, Fraser C VL et al. Effectiveness and efficiency of guideline dissimination and implementation strategies. Heal technol assess. 2004;8(iii-72).

27. Heneghan C, Perera R, Mant D GP. Hypertension guideline recommendations in general practice: awareness, agreement, adoption, and adherence. Br J Gen Pr. 2007;57(545):948-52.

28. Hagemeister J, Schneider CA, Barabas S, Schadt R, Wassmer G MG et al. Hypertension guidelines and their limitations - the impact of physicians' compliance as evaluated by guideline awareness. $J$ Hypertens. 2001;19(11):2079-86.

29. Legido-Quigley H, Panteli D, Mckee M, Busse R, Car J, Genet N, et al. HOME CARE ACROSS EUROPE CURRENT STRUCTURE AND FUTURE CHALLENGES The editors Clinical Guidelines for Chronic Conditions in the European Union Observatory Studies Series. Observatory Studies Series. 2010.

30. Prugger C, Heuschmann PU KU. Epidemiologie der Hypertonie in Deutschland und weltweit. Herz. 2006;31(4):287-93.

31. Legorreta AP, Christian-Herman J, O'connor RD, Hasan MM, Evans R, Leung K-M. Compliance With National Asthma Management Guidelines and Specialty Care A Health Maintenance Organization Experience [Internet]. Available from: https://jamanetwork.com/

32. Ogden LG, He J, Lydick E WP. Long-term absolute benefit of lowering blood pressure in hypertensive patients. Accord to JNC VI risk Stratif H. 2011;35:539-543.

33. Ahmad N, Khan AH, Khan I, Khan A, Atif M. Doctors' Knowledge of Hypertension Guidelines Recommendations Reflected in Their Practice. Int J Hypertens. 2018;2018:11-3.

34. G.-C.Chan. et al. "Type2 diabetes mellitus with hypertension at primary health care level in Malaysia: are they managed according toguidelines?" Singapore MedicalJournal. 2007;46(3):127-31. 
35. Ahmad N, Hassan Y, Tangiisuran B, Loke O. Guidelines Adherence and Hypertension Control in an Outpatient Cardiology Clinic in Malaysia. 2012;11(July 2011):665-72.

36. S. J. Houlihan, S. H. Simpson AJC et al. "Hypertension treatment and control rates:chart review in an academic family medicine clinic,." Can Fam Physician. 2009;55(87):735-741.

37. Adhikari S, Mathiasen R, Lander L. Elevated blood pressure in the emergency department: lack of adherence to clinical practice guidelines. Blood pressure monitoring. 2016;21(1):54-8.

38. Ramli AS, Miskan M, Ng KK, Ambigga D, Nafiza MN, Mazapuspavina MY, et al. Prescribing of antihypertensive agents in public primary care clinics - is it in accordance with current evidence. Malaysian Fam Physician. 2010;5(1):36-40.

39. Ernst S. Hypertension guideline adherence of private practitioners and primary health care physicians in Pretoria. 2003;51-4.

40. Adedeji AR, Tumbo J, Govender I. Adherence of doctors to a clinical guideline for hypertension in Bojanala district, North-West Province, South Africa. African J Prim Heal Care Fam Med. 2015;7(1):1-6.

41. Victor Basopo et al. To what extent do prescribing practices for hypertension in the private sector in Zimbabwe follow the national treatment guidelines? Journal of Pharmaceutical Policy and Practice (2017) 10:37 DOI 10.1186/s40545-017-0125-7

42. Hiba Salah Abdelgadir. et al. Adherence of doctors to hypertension clinical guidelines in academy charity teaching hospital, Khartoum, Sudan. Abdelgadir et al. BMC Health Services Research (2019) 19:309 [available on: https://doi.org/10.1186/s12913-019-4140-z]

43. Rogers EM. Diffusion of innovations. 4th ed. New York,: The Free Press; 1995.

44. Mayers PM. Nurses' Experiences of Guideline Implementation in Primary Health Care Settings. 2010.

45. Karbach U, Schubert I, Hagemeister J, Ernstmann N. Physicians ' Knowledge of and Compliance. 2011;(June 2014).

46. Cuspidi C, Michev I, Meani S, Severgnini B, Sala C, Salerno M, et al. Awareness of hypertension guidelines in primary care: results of a regionwide survey in Italy. J Hum hyprtension. 2003;11:5417.

47. Hagemeister J, Schneider CA, Diedrichs H, Mebus D, Pfaff H, Wassmer G, et al. 5-year follow-up of the hypertension evaluation project (HEP ). 2008;7:1-7.

48. Adamu UG, Ibok IO, Abdullahi A, Ogundele IO. Knowledge, Attitude and Practice of Physicians in the Treatment of Hypertension in North-Central Nigeria. world J cardiovasscular Dis. 2014;4:251-6.

49. Jecker NS. Physician Compliance with Guidelines. 2017;(April).

50. T. Wae, C.Man, L.Tong, C. Wang CY et al. "are we evidence based in prescribing for hypertension?" Asian pacific J Fam Med. 2006;5(3).

\section{Appendix}

Appendix II is not available with this version. 
Figures

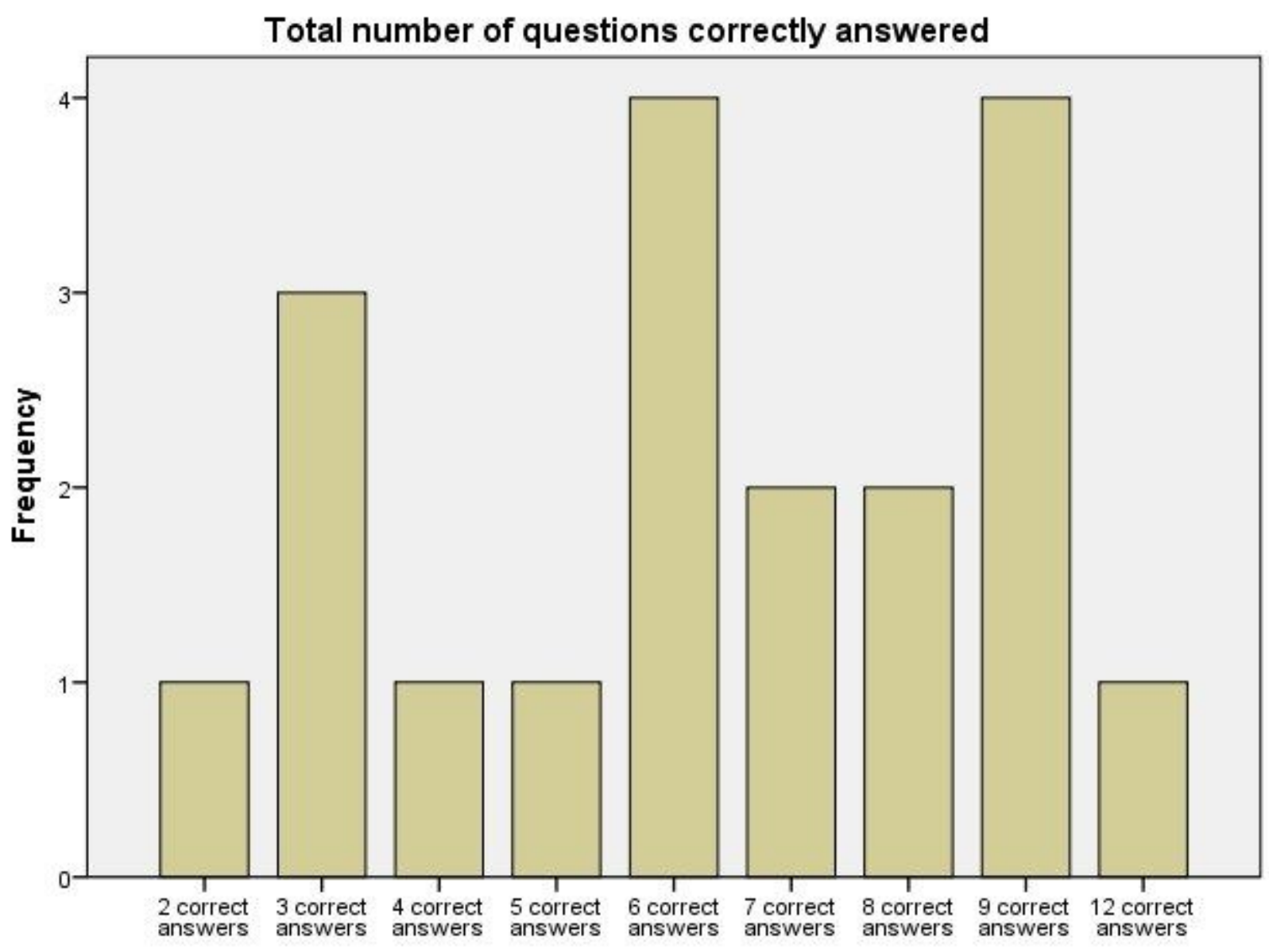

Total number of questions correctly answered

\section{Figure 1}

Absolute number of correct answers to the 12 questions on knowledge of HTN guideline in the 19 respondent health practitioners'. 


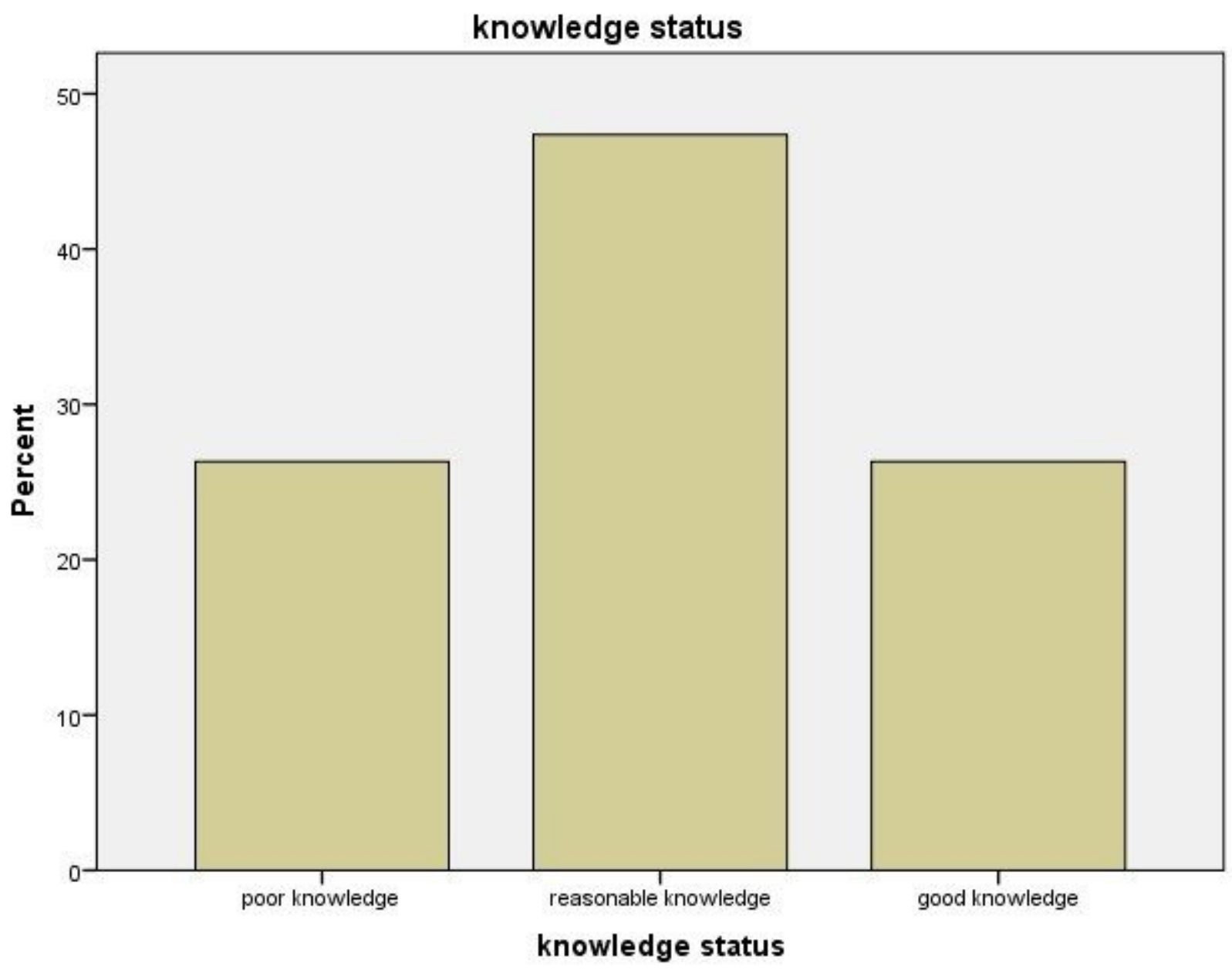

Figure 2

Health practitioners' knowledge status On the basis of criterion used for adequate knowledge 